\title{
WHAT ARE THE RESULTS OF ANATOMIC RECONSTRUCTION FOR UNSTABLE ACROMIOCLAVICULAR JOINT BY USING AN AUTOGENOUS SEMITENDINOSUS GRAFT?
}

\author{
Mohamed Mansour Elzohairy, MD. \\ Lecturer of orthopedic surgery, faculty of medicine Zagazig University.
}

\begin{abstract}
Acromioclavicular injury is considered as a common athletic shoulder injury. Many of these injuries can be treated nonoperatively but high-grade injuries can result in pain and loss of shoulder function. A numerous operative techniques have been proposed with recent renewed focus on the anatomy of the coracoclavicular ligaments which has led to anatomic reconstructive techniques that show promised results in biomechanical comparisons. These techniques involve reconstruction of the conoid and trapezoid ligaments through anatomically-based tunnels in the clavicle, with preservation of the distal clavicle that improves the biomechanical stability of this construct. The purpose of this study was to evaluate the results of anatomic reconstruction of unstable acromioclavicular joint by using an autogenous semitendinosus graft. All the patients gave the informed consent prior to being included into this study; the study was authorized by the local ethical committee and was performed in accordance with the Ethical standards of the 1964 Declaration of Helsinki as revised in 2000.

Keywords: Acromioclavicular joint, coracoclavicular ligament,reconstruction
\end{abstract}

\section{INTRODUCTION}

$\mathbf{T}$ he acromioclavicular joint is a diarthrodial joint between the clavicle and acromion and helps to link the shoulder to the axial skeleton. The joint is palpable on examination as a shallow depression between the end of the clavicle and the acromion. The articulation between these two bones can form at highly variable angles. The sides of this joint are covered with articular cartilage, with a fibrocartilagenous disk in between which is often referred to as a meniscal homologue. Stability to this joint is provided through two sets of ligaments the acromioclavicular (AC) and coracoclavicular (CC) ligaments. The acromioclavicular ligaments are

horizontally directed and function to control horizontal (anteroposterior) stability for the aromioclavicular joint. ${ }^{1,2}$ In addition to the joint capsule, the acromioclavicular ligaments intimately surround the joint and are divided into superior, inferior, anterior, and posterior components. The

superior ligament is the largest of the four components and is the primary acromioclavicular joint stabilizer for normal daily activities. The acromioclavicular ligaments attach to the clavicle approximately $1.5 \mathrm{~cm}$ from the joint. Excision of $1.5 \mathrm{~cm}$ or more of the distal clavicle may result in disruption of the acromioclavicular ligaments and lead to horizontal instability of the acromioclavicular joint. The coracoclavicular ligaments are the stronger, more vertically oriented ligaments that have two components, the conoid and trapezoid ligaments. The conical shaped conoid ligament is more medial and is the most important ligament for support against injuries and superior (vertical) displacement of the clavicle in relation to the acromion. ${ }^{3,4}$ This ligament runs from the conoid tubercle of the clavicle to the base of the coracoid process. The more laterally based trapezoid ligament lies anterior and lateral to the conoid tubercle and inserts more laterally on the base of the coracoid. This ligament primarily functions to resist compression across the acromioclavicular joint. The average insertional distance of the conoid ligament from the acromioclavicular joint is 28.9 $\mathrm{mm}$ in females and $33.5 \mathrm{~mm}$ in males. The insertional distances for the trapezoid ligament are 16.1 and $16.7 \mathrm{~mm}$ from the joint in females and males, respectively. The clinical relevance in these distances is found in the facts that a resection

of less than $11 \mathrm{~mm}$ does not violate the trapezoid ligament insertion and a resection of less than 24 $\mathrm{mm}$ should not disrupt the insertion of the conoid ligament in either gender in $95 \%$ of patients. ${ }^{5}$ Minimal motion, between $5^{\circ}$ and $8^{\circ}$, occurs through the acromioclavicular joint. ${ }^{6}$ This is due in part to the synchchronous motion between the clavicle and scapula. Clinically this is supported, with acromioclavicular joint fusions resulting in little dysfunction. ${ }^{5,6,7}$

\section{PATIENTS AND METHODS}

Thirteen patients' 9 males and 4 females with mean age of 35 years (24-50 years) were treated for unstable acromioclavicular joint (rockwood type IV) ${ }^{8}$ by anatomic reconstruction of unstable acromioclavicular joint with using an autogenous semitendinosus graft at Zagazig University Hospitals. The right shoulder was affected in six patients and the left shoulder was injured in seven. The causes of injury were traffic accidents in 3 patients and falling in ten. The mean period from injury to surgery was 6 days (range, 3-14 days). Clinical evaluation was performed using the American Shoulder and Elbow Surgeons shoulder score $^{9}$, after a mean follow-up time of 36 months. 
Preoperative and postoperative radiographs were compared.

\section{Operative technique: ${ }^{10,11}$}

Under general anesthesia with the patient in a beach chair position. The incision is centered in line with Langer's lines (vertical saber-type) approximately $1.5 \mathrm{~cm}$ medial to the AC joint and should be slightly angled to allow access laterally to the CA ligament and medially to the coracoid process. Beginning at the posterior aspect of the distal clavicle, the incision extends anteriorly to the coracoid process. Subcutaneous skin flaps are elevated to allow placement of a self-retaining retractor (figure1). The underlining deltoid fascia is carefully identified and the deltoid can be either elevated as a flap or lifted off in its entirety. We prefer to take the deltoid down with a "hockey stick" incision to visualize the coracoid process. The length of the deltoid elevation incision is made over the mid portion of the clavicle with electrocautery and extends laterally beyond the meniscal homologue to the edge of the acromion. Typically, the injury to the AC joint is readily apparent. The acromioclavicular ligaments are split from medial to lateral and are preserved to repair following the reconstruction. Once a periosteum flap has been elevated with electrocautery and elevators, a tagging suture is placed in the medial-most aspect of the periosteum flap. Just medial to the tagging stitch, the incision is carried inferiorly to complete the "hockey stick" elevation of the deltoid and allow visualization of the coracoid. The tagging suture allows accurate reattachment of the deltoid on closure. Once the deltoid has been lifted off the clavicle, the plane just superficial to the CA ligament should be identified and excess soft tissue removed to define the boarders of the ligament. To fully visualize the coracoacromial ligament, dissection must be performed laterally. Then in the same time another team takes and prepares the autogenous semitendinosus graft (figure 2). The fascia is incised along the length of the distal clavicle and subperiosteally dissected anteriorly and posteriorly. At this point, the distal clavicle can generally be reduced into the AC joint. If necessary, soft tissue debridement to include excision of the fibrocartilaginous disc within the joint capsule may be performed to aid in reduction. The dissection also is carried inferiorly to the coracoid base to allow passage of the graft inferior to the coracoid. At approximately $45 \mathrm{~mm}$ from the $\mathrm{AC}$ articulation, a guide pin is placed for the conoid tunnel and a 5.5 $\mathrm{mm}$ cannulated reamer is used to drill the tunnel. Then, approximately $15 \mathrm{~mm}$ lateral to that on the anterior border of the clavicle, the trapezoid tunnel is drilled again using a $5.5 \mathrm{~mm}$ reamer. The graft is passed inferior to the coracoids process and crossed over itself before being passed into the bone tunnels previously created in the clavicle. This cruciate graft position (Figures 3- a,b,c) approximates the origin of the ligaments on the coracoid process without the fracture risk associated with potting the grafts into the small coracoids process. Reduction of the AC joint is confirmed, and the graft is tensioned prior to being tied together with number 2 ethibond suture over the superior surface of the clavicle (Figure 4). We do not resect the distal clavicle but routinely excise fibrous tissue that can block reduction of the clavicle into the AC joint. Then we routinely used two key wires as temporary fixation which removed three weeks later on .This adjunct of an AC reconstruction is critical to restore the anteroposterior stability, which may lead to failure of an isolated CC reconstruction. Once the reconstructed ligaments are fixed, the adequacy of the reduction is confirmed by either direct view or fluoroscopy if necessary (figures 5$\mathrm{a}, \mathrm{b}, \mathrm{c}$ ) and (figures 6- a,b) . The AC joint capsule is repaired if present, and the deltotrapezial fascia is closed with absorbable suture. The patient is placed in a shoulder immobilizer before waking from general anesthesia. We currently use a standard shoulder immobilizer.

\section{REHABILITATION}

Postoperative rehabilitation consists of a program designed to initially protect the reconstruction, which then progresses to preserve shoulder range of motion (ROM) and eventually to regain strength and flexibility, allowing patients to return to physical activity. Strict compliance with the chosen rehabilitation protocol is critical to the success of acromioclavicular reconstruction. Patients frequently feel significant symptomatic relief from being operatively stabilized and are often inclined to aggressively pursue an active lifestyle, which can compromise graft incorporation and may lead to failure of the reconstruction. At our institution, patients are maintained in a shoulder immobilizer for a total of 6 weeks. Week 1 focuses on pain control and preservation of wrist and elbow active range of motion (AROM). Patients may take off their sling during physical therapy to perform supported pendulum exercises. Weeks 2--6 are focused on active assisted range of motion (AAROM). Physical therapy during this period consists of AAROM exercises with flexion up to 90 degrees, abduction to 60 degrees and external rotation as 
tolerated. The focus of weeks 6 through 12 are progressively increased ROM. At

3 months, patients are allowed to begin gentle strengthening. At 4-6 months, strength training is increased as well as sport-specific rehabilitation. Return to duty or sports are allowed at 6 months.

\section{RESULTS}

The mean American Shoulder and Elbow Surgeons shoulder score improved from $37+/-5$ points to $95+/-4$ points. The radiologic measurements showed a mean coracoclavicular distance of $11.3+/-4 \mathrm{~mm}$ increasing to $11.7+/-3$ $\mathrm{mm}$ under stress. One case suffered from superficial infection which treated successfully with oral antibiotic and repeated dressing. The greatest complication was failure of the reconstruction with recurrent deformity because of poor patient compliance with activity restrictions in the early postoperative period which happened in three patients $(23.07 \%)$.

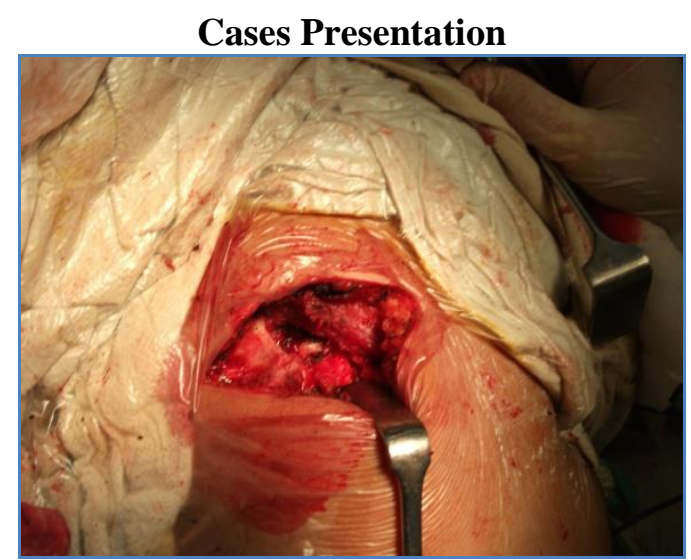

Figure (1): the incision is centered in line with Langer's lines (vertical saber-type) approximately $1.5 \mathrm{~cm}$ medial to the AC joint and should be slightly angled to allow access laterally to the CA ligament and medially to the coracoid process.

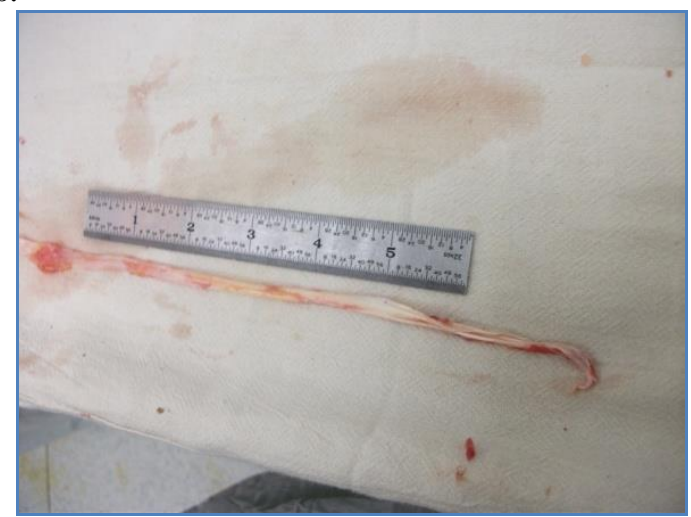

Figure (2): preparations of the autogenous semitendinosus graft.

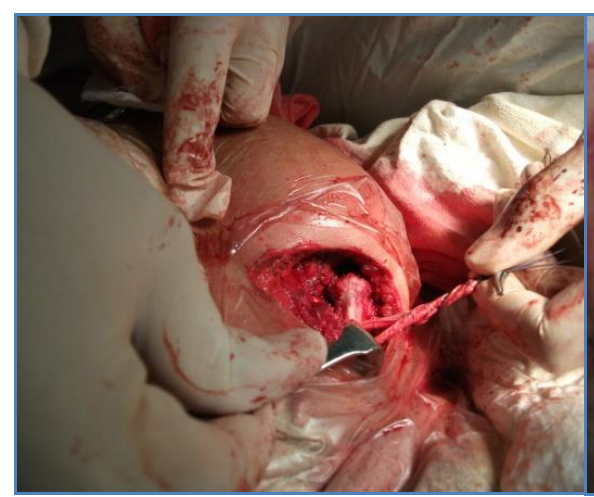

Figure 5 (a)

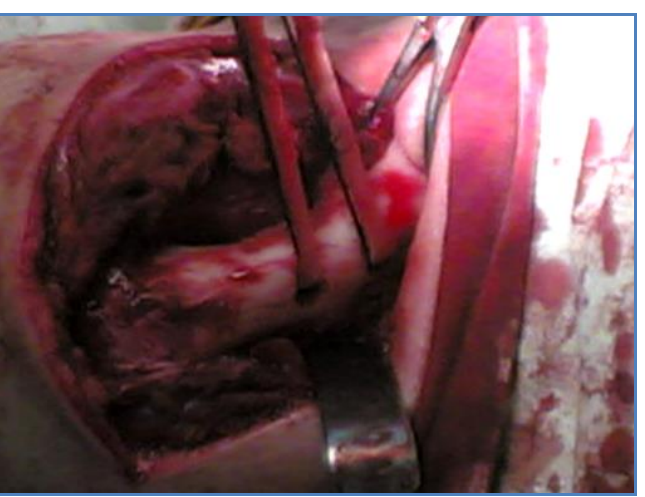

Figure 5 (b)

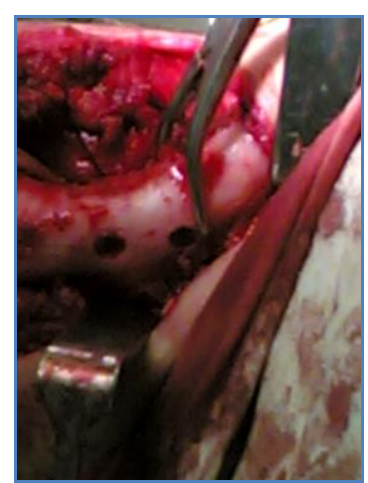

Figure 5 (c)

Figure $(3-a, b, c)$ : The graft is passed inferior to the coracoids process and crossed over itself before being passed into the bone tunnels previously created in the clavicle. This cruciate graft position approximates the origin of the ligaments on the coracoid process without the fracture risk associated with potting the grafts into the small coracoids process. 


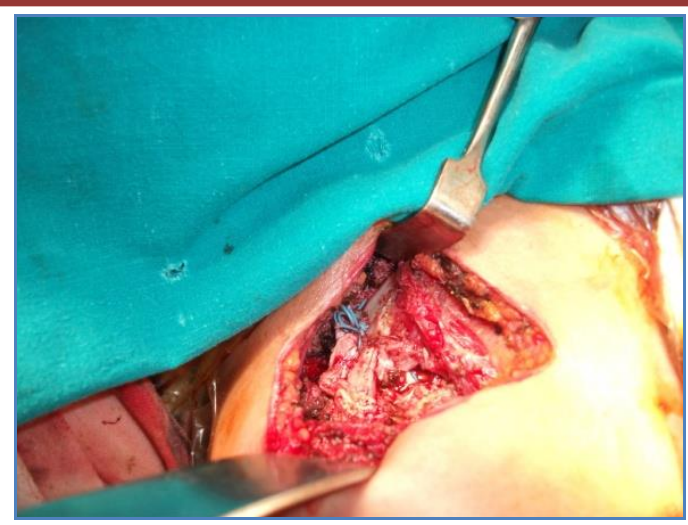

Figure (4): Reduction of the AC joint is confirmed, and the graft is tensioned prior to being tied together with number 2 ethibond suture over the superior surface of the clavicle.

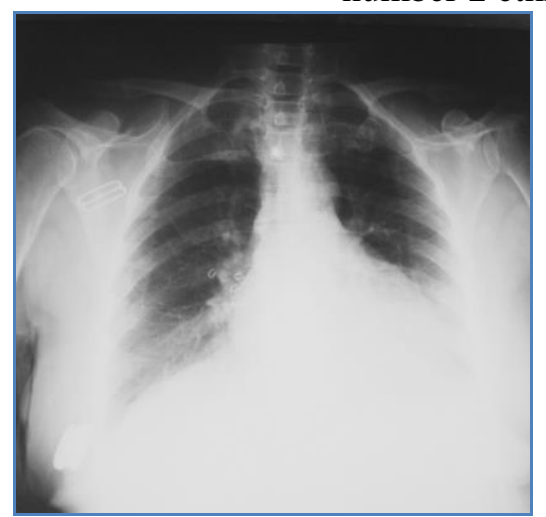

Figure 5 (a)

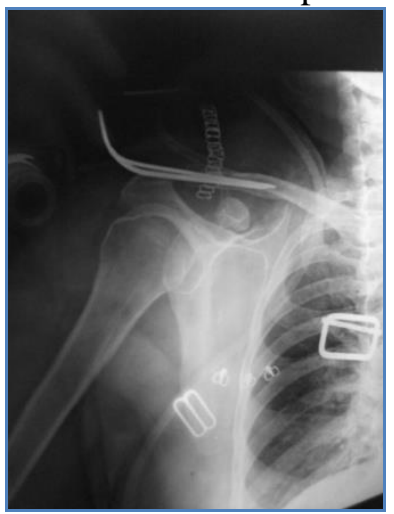

Figure 5 (b)

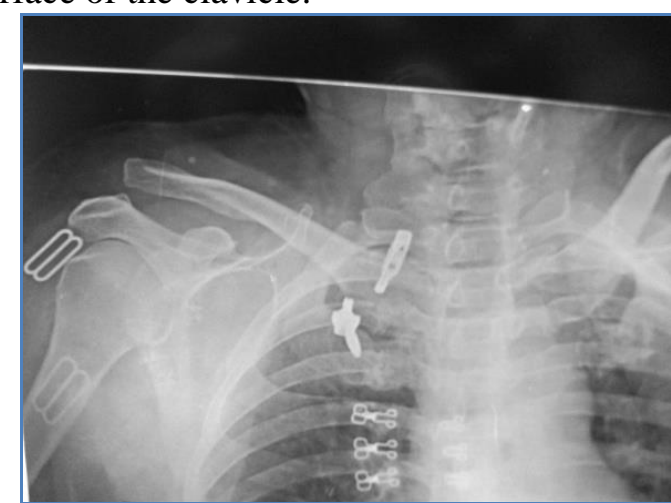

Figure 5 (c)

Figures $(5-a, b, c)$ : fifty years old female with right acromioclavicular dislocation (rockwood type IV) ${ }^{8}$ showing anteroposterior Zanca (cephaled $10^{\circ}$ ) views preoperative (a) and postoperative (b) after reduction with key wires fixation, (c) after 36 months fellow up.

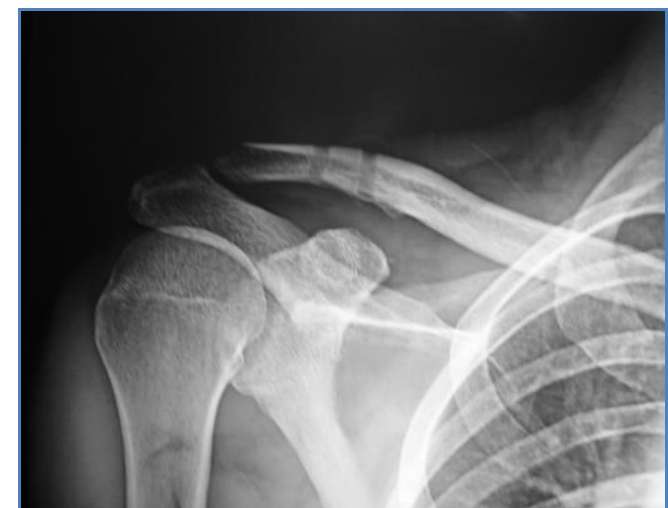

Figure 6 (a)

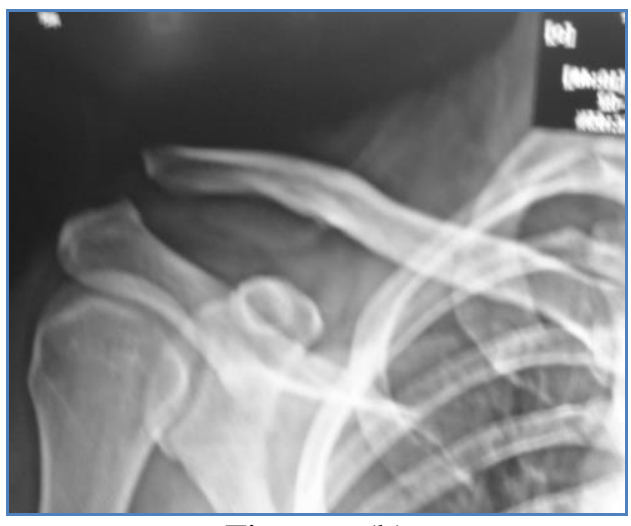

Figure $6(b)$

Figures (6-a, b): twenty four years old male with right acromioclavicular dislocation (rockwood type IV) ${ }^{8}$ showing anteroposterior Zanca (cephaled $10^{\circ}$ ) views preoperative (a) and postoperative (b) after 24 months fellow up.

\section{DISCUSSION}

The goal of AC joint reconstruction, when indicated, is to achieve an anatomic reconstruction that best restores both the restraint to vertical as well as anteroposterior translation of the clavicle at the $\mathrm{AC}$ joint. $^{7}$ There has some interest in primary or delayed excision of the distal clavicle (ie, Mumford procedure) in a symptomatic AC joint after dislocation. Although there has been success with this technique done open as well as arthroscopically, it must be reversed for patients in whom the CC ligaments are intact and there is no concomitant instability. ${ }^{12}$ When horizontal or vertical instability exists, results are compromised because this technique does not address instability and may accentuate it. ${ }^{\mathbf{2}}$ Numerous procedures 
have been described to treat the unstable AC joint. Technical options have included primary fixation at the $\mathrm{AC}$ joint (K-wires, hook plate), fixation between the coracoids and clavicle (CC screw, suture anchor, suture loop), dynamic muscle transfer, and ligamentous reconstruction. The traditional ligament reconstructive technique has been the Weaver-Dunn AC ligament transfer. ${ }^{7,} 11$ Multiple studies have examined the biomechanical results of various reconstructive techniques, and several conclusions can be drawn from them. A study by Jari et al ${ }^{13}$ suggests that surgical techniques that preserve the articulating surface of the clavicle at the AC joint lead to lower joint contact forces and are preferred. CA ligament transfer, which has been the most popular method for treating complete chronic AC dislocations, has just $25 \%$ of the strength of the intact CC complex and allows far greater primary and coupled translation than does the intact AC joint. $^{7}$ Augmentation of this repair and reconstruction with various suture loops and suture anchors can increase the construct strength and ultimate load to failure while reducing primary and coupled translation. Screw fixation may also be considered. Although the bicortical Rockwood screw has the highest tensile strength and stiffness with the lowest elasticity, these properties may be responsible for its high complication rate, including screw breakage and pullout. $^{7} \quad$ Fukuda et al. ${ }^{14}$, Dawson et al., ${ }^{15}$ Mazzocca et al. ${ }^{16}$ in their biomechanical studies concluded that each of the ligamentous structures surrounding the AC joint provides stability depending on the forces and directions of the loads. Therefore, operative procedures that allow the greatest number of structures to remain intact will provide superior strength after healing. The authors also noted that some procedures, such as distal clavicular resection, may not allow this to occur. They also concluded that for optimal operative repair the strategy should include reconstruction of both the AC joint capsule and the CC ligaments. While no clear consensus exists, recent anatomic and biomechanical research suggests that an anatomical reconstruction of the $\mathrm{CC}$ ligaments may provide a superior clinical outcome. ${ }^{11}$ In 2006; Mazzocca et al. ${ }^{17}$ published the results of a biomechanical evaluation of an anatomical CC ligament reconstruction. In their study, they randomly placed 42 fresh-frozen cadaveric shoulders into three operative groups: arthroscopic reconstruction, anatomical $\mathrm{CC}$ ligament reconstruction and a modified Weaver-Dunn procedure. In their study, they found that the mean anterior, posterior and superior translation of an intact specimen after a 70-N load was applied was 9.82_4.51, 7.39_4.16 and 5.63_2.14mm, respectively. After reconstruction using the described techniques, they determined that the Weaver-Dunn method demonstrated a significant increase in posterior translation: $11.17 \_4.60 \mathrm{~mm}$ $(\mathrm{P} 1 / 40.0315)$. The arthroscopic technique resulted in no change in translation in any plane when compared with the intact specimen. Anatomic CC ligamentous reconstruction demonstrated decreased posterior and superior translation when compared with the intact specimen. The authors concluded that "the anatomical CC reconstruction has less anterior and posterior translation and more closely approximates the intact state, restoring function of the $\mathrm{AC}$ and $\mathrm{CC}$ ligaments". ${ }^{17}$ Tauber et al. ${ }^{18}$ recently published the results of their prospective comparison between anatomical CC ligament construction using an autogenous semitendinosus graft and reconstruction using the modified Weaver-Dunn technique. In their study, they assigned 12 patients to the Weaver-Dunn group and 12 patients to the semitendinosus group. Patients were followed for a mean of 37 months and were evaluated using the American Shoulder and Elbow Surgeons (ASES) shoulder score and the Constant score. At followup, patients in the semitendinosus group demonstrated significant improvements in both their ASES shoulder scores and Constant scores. The authors concluded that CC ligamentous reconstruction using semitendinosus autograft resulted in significantly better outcomes when compared to the modified Weaver-Dunn technique. ${ }^{18}$ In our study we had good results in our cases with the technique of anatomic reconstruction for the unstable acromioclavicular joint (rockwood type IV) ${ }^{8}$ by using an autogenous semitendinosus graft. The failure of this technique was mainly attributed to poor patient compliance with activity restrictions in the early postoperative period which happened in three patients. The importance of patient good compliance with activity restrictions is to allow time for the graft to be revascularized and gain strength again by the creeping substitution because the graft will be atrophied and the bone tunnel will be widened which became slackened within the bone tunnel with upward displacement of the clavicle followed by subsequent return of acromioclavicular joint instability. Gracia et al, ${ }^{11}$ using the procedure in which The conoid ligament is fixed to the clavicle using a $5.5 \times 8 \mathrm{~mm}$ PEEK tenodesis screw (Arthrex, Naples,FL) and then after reduction of the AC joint is confirmed, and the graft is tensioned prior to fixation of the trapezoid ligament with another tenodesis screw as a 
method to prevent this complication but, they still had numbers of failures of the reconstruction with recurrent deformity because of poor patient compliance with activity restrictions in the early postoperative period. In the future this modification will be fully investigated by us and compared with the other technique.

\section{CONCLUSION}

The semitendinosus graft is a biomechanically strong, biologic option for reconstruction of the coracoclavicular ligaments especially in unstable acromioclavicular joint (Rockwood type IV). The Anatomical acromioclavicular reconstruction recreates the strength of the native acromioclavicular joint.

\section{REFERENCES}

1. DePalma AF: The role of the disks of the sternoclavicular and the acromioclavicular joints. Clin Orthop13:7-12, 1959.

2. Salter EG, Jr, Nasca RJ, Shelley BS: Anatomical observations of the acromioclavicular joint and

supporting ligaments. Am J Sports Med 15:199-206, 1987.

3. Urist MR: Complete dislocations of the acromioclavicular joint: The nature of the traumatic lesion and effective methods of treatment with analysis of forty-one cases. J Bone Joint Surg 28A:813-837, 1946.

4. Fukuda K, Craig EV, An K, Cofield RH, Chao EYS: Biomechanical study of the ligamentous system of the acromioclavicular joint. J Bone Joint Surg 68A:434-440, 1986.

5. Flatow EL: The biomechanics of the acromioclavicular, sternoclavic-ular and scapulothoracic joints. Instr Course Lect 42:237245, 1993.

6. Ponce AB, Millet JP, Warner JJP: Acromioclavicular joint instability reconstruction indication and technique. Operative Techniques in Sports Medicine, Vol 12, No 1 (January), pp 35-42, 2004.

7. Simovitch R, Sanders B, Ozbaydar M, Lavery K, Warner PJJ: Acromioclavicular joint injuries: diagnosis and management. J Am Acad Orthop Surg 17:207-219, 2009.
8. Williama GRJr, Nguyen VD, Rockwood CA Jr: Classification and radiographic analysis of acrromioclavicular dislocations. Appl radiol, 18:2934, 1989.

9. Richard RR, An K, Bigliani UL, Friedman JR, Gartsman MG, Gristina GA, Lannotti PJ, Mow CV, Sidles AJ, Zuckerman DJ: A standardized method for the assessment of shoulder function. J. Shoulder Elbow Surg Volume 3, Number 6, 347-352, 1994.

10. Mazzocca AD, Santangelo SA, Johnson ST, et al. A biomechanical evaluation of an anatomical coracoclavicular ligament reconstruction. Am J Sports Med.; 34:236--246. 2006.

11. Gracia JE, Owens DB: Anatomic approach to reconstruction of the unstable acromioclavicular joint. Current orthopaedic practice, Volume 21, Number 1, January/February 2010.

12. Corteen DP, Teitge RA: Stabilization of the clavicle after distal resection: A biomechanical study. Am J Sports Med, 33:61-67, 2005.

13. Jari R, Costic RS, Rodosky MW, Debski RE: Biomechanical function of surgical procedures for acromioclavicular joint dislocations. Arthroscopy, 20:237-245, 2004.

14. Fukuda K, Craig EV, An KN, et al. Biomechanical study of the ligamentous system of the acromioclavicular joint. J Bone Joint Surg. 68:434-440, 1986.

15. Dawson PA, Adamson GJ, Pink MM, et al. Relative contribution of acromioclavicular joint capsule and coracoclavicular liga-ments to acromioclavicular stability.J Shoulder Elbow Surg. 18: 237-244, 2009.

16. Mazzocca AD, Spang JT, Rodriguez RR, et al. Biomechanical and radiographic analysis of partial coracoclavicular ligament injuries. Am J Sports Med. 36:1397-1402, 2008.

17. Mazzocca AD, Santangelo SA, Johnson ST, et al. A biomechani-cal evaluation of an anatomical coracoclavicular ligament reconstruction. Am J Sports Med. 34:236-246, 2006.

18. Tauber M, Gordon K, Koller $\mathrm{H}$, et al. Semitendinosus tendon graft versus a modified Weaver-Dunn procedure for acromio-clavicular joint reconstruction in chronic cases: a prospective comparative study. Am J Sports Med.37:181-190, 2009. 


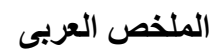

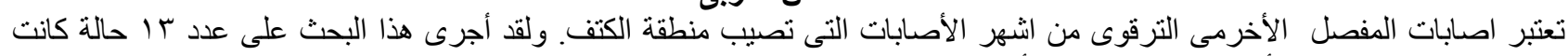

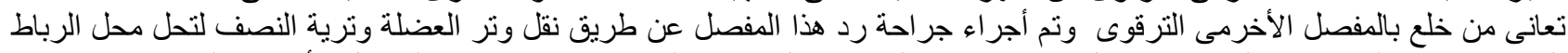

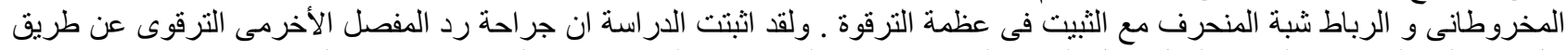

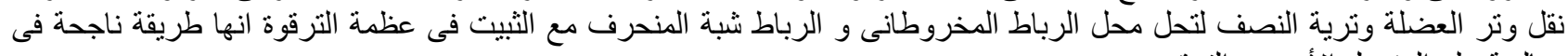
معالجة خلع المفصل الأخرمى الترقوى . 